\title{
DESIGUALDADES SOCIOECONÓMICAS Y SUS IMPLICANCIAS EN EL ESTADO DE SALUD. ALGUNOS INDICADORES APLICADOS A LA PROVINCIA DE BUENOS AIRES
}

\author{
María Florencia Arnaudo*
}

recibido: septiembre 2014 - aceptado: marzo 2015

\section{INTRODUCCIÓN}

En las últimas décadas diversas investigaciones muestran la relación entre los bajos ingresos y la pobreza de la población y su déficit de salud. En efecto, en los países que han alcanzado menor nivel de desarrollo se evidencian peores resultados sanitarios que en los de mayor desarrollo y, dentro de cada país, las personas de bajos ingresos tienen más problemas de salud que las de altos ingresos. En este sentido, los grupos de población que presentan las condiciones socioeconómicas y culturales más desfavorables tienen menor acceso a los servicios de salud que a su vez, en general, son de baja calidad. Esto redunda en una prevalencia de enfermedades crónicas tratables y previsibles e incluso tasas de mortalidad mayores (Dallolio, 2012; Wagstaff, 2002).

La literatura especializada sostiene que la tasa de mortalidad infantil $\left(\mathrm{TMI}^{1}\right)$ -que indica el riesgo de muerte para los niños menores de un año-, no sólo señala la ocurrencia del fenómeno biológico, sino que sugiere condiciones de vivienda, alimentación, educación, saneamiento ambiental y otros factores que caracterizan el estilo y la calidad de vida de la población. Por lo tanto, dado que la TMI puede relacionarse con el bienestar y el cuidado general de la atención de la salud puede ser considerada una variable indicadora del nivel de calidad de vida y utilizarse

\footnotetext{
* IIESS-CONICET-UNS

1 La tasa de mortalidad infantil es un indicador demográfico que señala el número de defunciones de niños menores a un año cada mil nacimientos vivos registrados. La consideración del primer año de vida para establecer el indicador de la mortalidad infantil se debe a que este año es el más crítico en la supervivencia del ser humano. Además cuando se alcanza el primer año de vida, las probabilidades de supervivencia aumentan significativamente.
} 
para relacionar el estado de salud de la población con el nivel de desarrollo de un país con (WHO, 2007; The World Bank, 2007; Reidpath y Allotey, 2003).

En Argentina, en la década del ' 90 se llevó a cabo un proceso de ajuste económico caracterizado por la reforma del estado que implicó terminar con el Estado empresario, regulador, e intervencionista para pasar a un Estado reducido cuyo único objetivo consistía en cumplir lo encomendado en la Constitución Nacional. En este periodo, a pesar del crecimiento del PBI, la desigualdad en la distribución del ingreso, de la riqueza y de los bienes culturales se profundizó. Posteriormente, luego de la crisis sociopolítica del 2001, la actividad económica se recuperó significativamente. Esta recuperación fue acompañada por políticas activas del Estado que perseguían la inclusión de todos los habitantes y la construcción de un orden social más justo y solidario (González y Peranovich, 2012).

En los últimos años, diversos factores sociales, ambientales, culturales, y tecnológicos junto con la ampliación de la cobertura de servicios de salud ${ }^{2}$ y programas de salud pública permitieron que la TMI cayera de 9,1 puntos porcentuales $^{3}$, tendencia decreciente que se mantiene hasta la actualidad. Sin embargo, se observa que los logros alcanzados no se distribuyen uniformemente en toda la población, es decir se evidencian diferencias en el estado de salud.

En la Provincia de Buenos Aires, en particular, el Ministerio de Salud, reconoce que si bien en la última década disminuyó la TMI, siendo en el año 2011 $11,7 \%$, todavía es necesario obtener mejores resultados con la finalidad de lograr las metas delineadas en los Objetivos de Desarrollo del Milenio. Estas metas consisten en alcanzar, por un lado, en el año 2015 una tasa de mortalidad infantil de 7,5\%, y, por otro, que las tasas correspondientes a los partidos que la componen no supere el dígito y, a su vez, que la brecha entre las mismas no sea superior al 25\% (Ministerio de Salud de la Provincia de Buenos Aires, 2008).

En este contexto, el objetivo de este trabajo es verificar si la disminución de la TMI se distribuyó igualitariamente en la Provincia a partir de la aplicación de diversos indicadores relativos al análisis de desigualdad en el estado de salud, tales como, el índice de efecto, el índice relativo de desigualdad, el índice de desigualdad

2 Esta ampliación se llevó a cabo, en parte, a través de la formación del Plan Federal que promueve la Atención Primaria de la Salud, mejorar el acceso y la utilización de los servicios de salud, satisfacer la demanda de la población en relación a los servicios de salud adaptándose a las necesidades y aspiraciones de la misma (Rossen, et al, 2007).

3 En el año 1988 fue $25,6 \%$ descendiendo a $16,5 \%$ en 2003. 
de la pendiente, el riesgo atribuible poblacional, y la curva de Concentración e Índice de Concentración en la Provincia de Buenos Aires para el período 2007-2011.

\section{METODOLOGÍA}

En pos de lograr el objetivo se realiza un estudio ecológico en el cual la unidad de análisis está compuesta por los partidos de la Provincia agrupados en quintiles de población como se detalla más adelante. Se trata de un estudio de tipo transversal, es decir que se estudia la relación entre el estado de salud y el nivel socioeconómico de la población en un momento dado.

Para el cálculo de los diversos indicadores se utiliza como variable indicativa del status de salud la TMI promedio para los años 2007-2011 para cada partido de la Provincia ${ }^{4}$. Los datos fueron extraídos de las publicaciones editadas por la Dirección de Información Sistematizada del Ministerio de Salud Provincial (DEIS, 2014).

El nivel socioeconómico de cada partido se refleja a través del porcentaje de hogares con Necesidades Básicas Insatisfechas (NBI) ${ }^{5}$, a partir de la información el Censo Nacional de Población, Hogares y Viviendas realizado en el año 2010 (INDEC, 2010). Este indicador utilizado para medir de manera directa la pobreza considera que un hogar tiene NBI si presenta al menos uno de los siguientes indicadores de privación: hacinamiento crítico (más de 3 personas por cuarto), vivienda de tipo inconveniente (pieza de inquilinato, vivienda precaria u otro tipo), hogares que habitan en viviendas que no tienen retrete o tienen retrete sin descarga de agua, hogares que tienen algún niño en edad escolar que no asiste a la escuela, hogares que tienen 4 ó más personas por miembro ocupado y en los cuales el jefe tiene bajo nivel de educación (sólo asistió dos años o menos al nivel primario) (Feres y Mancero, 2001).

Para el análisis de las desigualdades, la población de los partidos de la Provincia de Buenos Aires se agrupa en quintiles de población ordenados, en orden ascendente según el porcentaje de hogares con NBI. Así, el primer quintil incluye al 20\% de la población con el menor porcentaje de NBI, es decir, la de mayor nivel

4 Se recurre al promedio, por un lado, para compensar el bajo número de sucesos en algunos de los partidos y, por otro, para morigerar las variaciones anuales.

5 Si bien la pobreza, puede ser medida utilizando el nivel de ingresos del jefe de familia o del grupo familiar, se recurre a las NBI debido a que el nivel de ingreso, no está disponible. Además, el NBI, se recoge dentro de la información censal por lo tanto está disponible para todos los partidos. 
socioeconómico. Mientras que el último quintil, agrupa al 20\% de la población con el menor nivel socioeconómico, esto es, el mayor porcentaje de hogares con NBI. Luego de este agrupamiento para cada quintil se calculó el promedio del porcentaje de hogares con NBI, y la TMI a partir del total de niños menores de un año fallecidos y el total de niños nacidos vivos en el período 2007-2011.

La relación entre las desigualdades de salud de la población y los factores socioeconómicos está siendo investigada por la Epidemiología desde sus orígenes (Berkman y Kawachi, 2000). Estas desigualdades pueden valorarse a partir de diversos indicadores que intentan captar el efecto de la situación socioeconómica sobre las condiciones de salud o su impacto ${ }^{6}$, tanto en términos relativos como absolutos, los cuales se describen brevemente a continuación 7 .

La Diferencia de tasas se calcula como la diferencia entre las tasas correspondientes a los grupos extremos, indica cuántos sucesos -muertes- se producen en el grupo menos favorecido en relación a las del grupo más favorecido.

El Riesgo Atribuible Poblacional (RAP) integra el grupo de las medidas de impacto y permite estimar el cambio que se produciría en el indicador de salud en el caso hipotético de que toda la población contara con el nivel socioeconómico del grupo más favorecido ${ }^{8}$.

Este indicador puede tomar valores entre 0 y 1 , reflejando la existencia de igualdad y perfecta desigualdad respectivamente?

El cálculo del RAP se realiza como sigue,

$\mathrm{RAP}=\underline{\mathrm{TMI}}$ general $-\mathrm{TMI}$ del grupo con mayor nivel socioeconómico

TMI general

6 Las medidas de impacto constituyen una fuente de información para la toma de decisiones y la formulación de políticas públicas destinadas a lograr la equidad debido a que consideran el estado real de la situación socioeconómica y pretenden estimar los cambios esperables en la condición de salud como resultado de posibles intervenciones, siendo esta su principal diferencia con las medidas de efecto. La relación entre los conceptos de efecto e impacto puede homologarse con la existente entre los conceptos de riesgo relativo y riesgo atribuible.

7 El análisis y descripción de estas medidas se realiza a partir de Schneider, et al (2002) y Kunst (1997), Szwarcwald, et al (2002), Alleyne, et al (2002) y Borrell, et al (2000)

8 Si el objetivo es medir la desigualdad con relación a la menor tasa encontrada, el grupo de referencia para el cálculo del RAP podría ser el grupo con el valor observado más bajo.

9 Esta definición supone una relación monótona entre el indicador socioeconómico y el indicador de salud que haría que el grupo con el mayor nivel socioeconómico sea el grupo con la menor tasa de morbilidad o mortalidad. 
El Índice de efecto describe las desigualdades entre todos los grupos de población. Para estimarlo es necesario realizar un análisis de regresión en el que se utiliza como variable dependiente la tasa de mortalidad y como variable independiente un indicador del nivel socioeconómico, en este caso el porcentaje hogares con NBI. La pendiente de la estimación obtenida, indica el valor del índice. A partir de su cálculo es posible estimar cómo y cuánto variará la tasa de mortalidad infantil cuando cambia la situación socioeconómica del grupo.

El índice de desigualdad de la pendiente es la pendiente de un modelo de regresión lineal en la cual, la variable dependiente es la TMI mientras que la variable explicativa es el rango promedio de los miembros de los grupos ordenados según el indicador socioeconómico. Dicho rango se denomina ridity, por definición adopta valores entre 0 (extremo inferior del ordenamiento socioeconómico) y 1 (extremo superior). Este índice ilustra cuánto cambia la TMI cuando la posición relativa del grupo cambia en una unidad.

Por último, la Curva de Concentración e Índice de Concentración es una herramienta gráfica, que compara la distribución de una variable con su distribución igualitaria. En el eje de las abscisas se representa la proporción acumulada de la población, ordenada según su nivel socioeconómico, y en el eje de las ordenadas la proporción acumulada de la variable de salud analizada. Si la salud se distribuye homogéneamente entre la población la curva se sitúa en la diagonal, mientras que cuanto más se aleja la curva de la diagonal mayor es el grado de desigualdad.

Para la elaboración de esta curva, por cuestiones metodológicas se utiliza como variable indicativa de la situación de salud el número de muertes de niños menores de un año en lugar de la TMI.

El coeficiente de Concentración es una medida resumen de la desviación de la curva de Concentración con respecto a la diagonal de igualdad. El valor del índice de Concentración dependerá de las características de la variable de salud considerada. Así, si se trata de una variable que favorece la salud el índice será positivo, con un rango entre [0,1], si el índice tiende a 0 menor desigualdad y en tanto se acerque a 1 mayor desigualdad. En cambio, si se trata de una variable negativa para la salud, el índice será negativo, con un rango entre $[-1,0]$. 


\section{RESULTADOS}

En el año 2001, en la Provincia aproximadamente murieron 12 niños menores de un año cada mil niños nacidos vivos. En el Conurbano, esta tasa fue levemente superior $(12,82 \%)$ que en el resto de las jurisdicciones $(11,45 \%)$, observándose una gran dispersión entre los partidos -en los extremos se encuentra Lobería con una TMI de 21,42\%o y Alberti de 4,51\%.

Para el análisis de las desigualdades en el status de salud, evaluadas mediante la TMI, asociadas a diferencias en el nivel socioeconómico se agrupó a la población de los diversos partidos en quintiles ordenados según el porcentaje de hogares con NBI, observándose una relación directa entre ambos. La TMI crece a medida que aumenta el porcentaje de hogares con NBI, es decir, a medida que los hogares sufren mayor grado de vulnerabilidad, presentando una diferencia de 3 puntos porcentuales entre el primer y quinto quintil (Tabla $\mathrm{N}^{\circ} 1$ ).

En el grupo de partidos con mayor porcentaje de hogares con NBI mueren aproximadamente 1.000 niños menores de un año más que en el grupo de partidos correspondientes al primer quintil. En este sentido, si todos los partidos de la Provincia tuvieran el mismo nivel socioeconómico que el grupo más aventajado, es decir el primer quintil, se evitarían el 17\% de las muertes infantiles (Tabla N²).

Tabla $N^{o}$ 1. Provincia de Buenos Aires: Tasa de Mortalidad Infantil según nivel socioeconómico

\begin{tabular}{ccc}
\hline Quintil & $\begin{array}{c}\text { \% Promedio de hogares } \\
\text { con NBI } 2010\end{array}$ & $\begin{array}{c}\text { TMI (\%) } \\
2007-2011\end{array}$ \\
\hline $1^{\circ}$ & 3,5 & 10,29 \\
$2^{\circ}$ & 5,3 & 11,82 \\
$3^{\circ}$ & 8,7 & 12,58 \\
$4^{\circ}$ & 11,2 & 13,17 \\
$5^{\circ}$ & 13,3 & 13,52 \\
\hline
\end{tabular}

Fuente: Elaboración Propia en base a INDEC y a Dirección de Información Sistematizada del Ministerio de Salud Provincial 
Tabla $\mathrm{N}^{\mathrm{o}} 2$. Diferencia de tasas y Riesgo Atribuible Poblacional

Diferencia de Tasas

RAP
$3,24 \%$

$17 \%$

Fuente: Elaboración Propia en base a INDEC y a Dirección de Información Sistematizada del Ministerio de Salud Provincial

Las medidas presentadas anteriormente sólo toman en cuenta los grupos extremos, ignorando los grupos no incluidos en la comparación. Esta limitación puede superarse a partir de la utilización de modelos de regresión. Así, el índice de desigualdad de la pendiente indica en el grupo de nivel socioeconómico más bajo $\left(5^{\circ}\right.$ Quintil) existe un exceso de mortalidad infantil de alrededor de 1.5 niños menores de un año por cada mil nacidos vivos en el grupo más favorecido ( $1^{\circ}$ Quintil).

El índice de efecto calculado utilizando para cada quintil de población el promedio de porcentaje de hogares con NBI como variable independiente y la TMI del período 2007-2011 como variable dependiente arroja un valor de 0,3. Esto indica que si disminuye en un punto porcentual la cantidad de hogares con NBI, la TMI también decrece en 0,3 defunciones por cada mil nacidos vivos.

Gráfico $\mathrm{N}^{\mathrm{o}}$ 1. Índice de Efecto entre la población de los partidos de la provincia de Buenos Aires agrupada de menor a mayor porcentaje de hogares con NBI y la mortalidad infantil

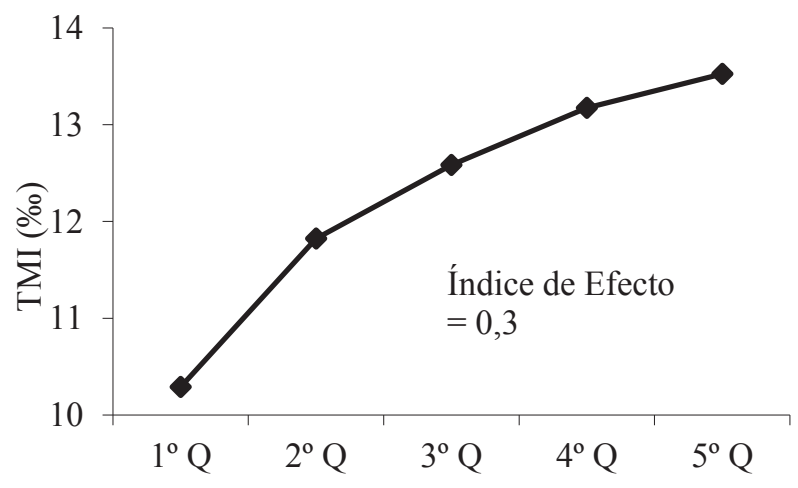

Fuente: Elaboración Propia en base a INDEC y a Dirección de Información Sistematizada del Ministerio de Salud Provincial 
Por último, el análisis de la curva de Concentración muestra que el 20\% de la población que reside en los partidos menos aventajados, es decir, con el peor nivel socioeconómico registra aproximadamente el $50 \%$ de las muertes de niños menores de un año. Mientras que en el grupo con mejor nivel socioeconómico ocurren un $1,82 \%$ de las muertes de niños menores a un año de la Provincia.

Gráfico No2. Curva de Concentración entre la población de los partidos de la provincia de Buenos Aires agrupada de menor a mayor porcentaje de hogares con NBI y la mortalidad infantil

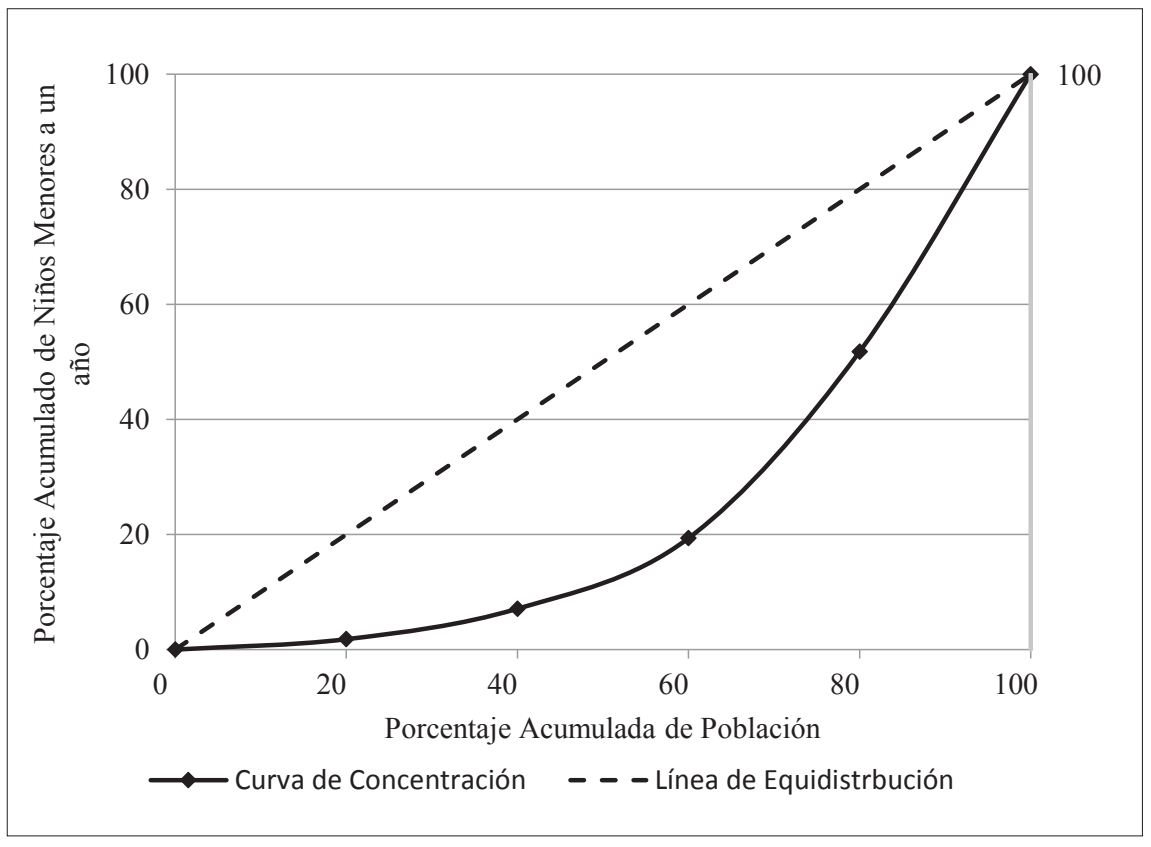

Fuente: Elaboración Propia en base a INDEC y a Dirección de Información

Sistematizada delMinisterio de Salud Provincial 


\section{CONCLUSIÓN Y DISCUSIÓN}

Mediante la utilización y comparación de diversos indicadores se evidencia la existencia de desigualdades significativas en la situación de salud de la población de la Provincia de Buenos Aires asociadas a la situación socioeconómica de la misma. Cabe destacar que se recurrió a distintas medidas de desigualdad, expresadas tanto en términos absolutos como relativos, debido a que cada una de ellas contempla diferentes puntos de vista, y por lo tanto permiten analizar el problema desde una perspectiva integral (Borrel, 2000).

No obstante, atribuir las diferencias en las condiciones de salud sólo a determinantes socioeconómicos medidos a partir de las necesidades básicas insatisfechas no es suficiente ya que existen otros factores -culturales, educacionales, medioambientales, de infraestructura- que influyen sobre las condiciones de salud que no están totalmente captados por dicho indicador (Szwarcwald, Bastos y Tavares de Andrade, 2000; Borrel, 2000). Por lo tanto, para una mejor comprensión de la dinámica de las diferencia de salud, es necesaria la construcción de una "red de causalidad capaz de representar no sólo las relaciones entre los factores socioeconómicos y el indicador de salud bajo análisis, sino también los efectos que intervienen en las acciones de salud, que, conjuntamente con los primeros, pueden afectar intensamente la respuesta" (Szwarcwald, Bastos y Tavares de Andrade, 2000).

La identificación y medición de las diferencias en las condiciones de salud y de vida constituye el primer paso para reconocer inequidades en salud, y por lo tanto, para el diseño y ejecución de acciones tendientes a disminuirlas y, eventualmente, eliminarlas (Alleyne, 2000).

\section{REFERENCIAS BIBLIOGRÁFICAS}

Alleyne G., Castillo-Salgado C., Schneider M., Loyola E., \&Vidaurre M. (2002). Overview of social inequalities in health in the region of the Americas, using various methodological approaches. Revista Panamericana de Salud Publica 12(6), 388-97.

Berkman L., \& Kawachi I. (Eds). (2000). Social Epidemiology. Oxford: Oxford University Press; 2000.

Borrell, C., Rué, M., Pasarín, M., Benach J., \& Kunst, A. (2000). La medición de las desigualdades en salud. Gaceta Sanitaria, 14(Supl 3), 20-33.

Dallolio L., Di Gregori V., Lenzi J., Franchino G., Calugi S., Domenighetti G., et 
al. (2012). Socio-economic factors associated with infant mortality in Italy: an ecological study. International Journal for Equity in Health, 11(1), 45-54. Dirección de Información Sistematizada del Ministerio de Salud Provincial. (DEIS). (2014). Hechos vitales y sus respectivas tasas segun region sanitaria 2007-2011. Recuperado de http://www.deis.gov.ar/

Feres, J. \& Mancero, X. (2001). El método de las necesidades básicas insatisfechas (NBI) y sus aplicaciones en América Latina. Chile: Naciones Unidas.

González, L. \& Peranovich, A. (2012). Años de esperanza de vida perdidos en Argentina, 2001-2010. Población y Salud en Mesoamérica, 10(1). Recuperada de http://ccp.ucr.ac.cr/revista/

Instituto Nacional de Estadística y Censo (INDEC). (2010). Censo Nacional de Población, Hogares y Viviendas 2010. Recuperado de http://200.51.91.245/ $\operatorname{argbin} / \mathrm{RpWebEngine.exe/PortalAction}$ \&MODE$=\mathrm{MAIN} \& \mathrm{BASE}=\mathrm{CPV} 2$ 010B\&MAIN=WebServerMain.inl

Kunst, A. (1997). Cross-national comparisons of socio-economic differences in mortality (tesis doctoral). Recuperada de repub.eur.nl/pub/18378/970917_ KUNST,\%20Anton\%20Eduard.pdf

Ministerio de Salud de la Provincia de Buenos Aires. (2008). Objetivos de desarrollo del milenio. Recuperado de: http://www.google.com.ar/url?sa=t\&rct=j\&q $=\&$ esrc $=$ s\&source $=$ web $\& c d=1 \&$ ved $=0$ CDEQFjAA\&url $=h t t p \% 3 \mathrm{~A} \% 2 \mathrm{~F} \%$ 2Fwww.ms.gba.gov.ar\%2FEstadodeSalud\%2Fobjetivosmilenio\%2FMilen io-2008.pps\&ei=83LCUMf1BY689gTFyIDIAQ\&usg=AFQjCNFC9XPn APMwytSYGqhehj7NeoRfpA\&sig2=h-W1XgoufjfURfvjarKnmg

Reidpath D, Allotey P. (2003). Infant mortality rate as an indicator of population health. Journal of Epidemiology \& Community Health, 57, 344-46.

Rossen M., Insaurralde M., Amarilla A., Lazaro C., Libertino M., \& Marconi E. (2007). Programa Federal de Capacitación de RRHH en Salud. Buenos Aires: TZE S.A.

Schneider M, Castillo-Salgado C, Bacallao J, Loyola E, Mujica O, Vidaurre M, et al. (2002). Métodos de medición de las desigualdades de salud. Revista Panamericana de Salud Pública/Pan American Journal of Public Health, 12(6), 398-415.

Szwarcwald C., Bastos F., \& Tavares de Andrade C. (2002). Medidas de desigualdad en salud: la discusión de algunos aspectos metodológicos con una aplicación para la mortalidad neonatal en el Municipio de Rio de Janeiro, 2000. Cadernos de Saúde Pública, 18(4), 959-970. Recuperado de http://www. scielo.br/scielo.php?script=sci_arttext\&pid=S0102-311X2002000400005

The World Bank. (2007). Facts and Figures from World Development Indicators 2007.Recuperado de http://siteresources.worldbank.org / DATASTATISTICS/Resources/reg_wdi.pdf 
Wagstaff A. (2002). Pobreza y desigualdades en el sector de la salud. Revista Panamericana de Salud Pública/Pan American Journal of Public Health, 11(5/6), 315-326.

World Health Organization. Ten statistical highlights in global public health. Recuperado de http://www.who.int/whosis/whostat2007_10highlights.pdf

(C) 2015 por los autores; licencia otorgada a la Revista Estudios Económicos. Este artículo es de acceso abierto y distribuido bajo los términos y condiciones de una licencia Atribución-No Comercial 3.0 Unported (CC BY-NC 3.0) de Creative Commons. Para ver una copia de esta licencia, visite http:// creativecommons.org/licenses/by-nc/3.0/ 